\title{
MODELOS LOGÍSTICOS PARA LA OPTIMIZACIÓN DEL TRANSPORTE DE RACIMOS DE FRUTO FRESCO DE PALMA DE ACEITE EN COLOMBIA
}

\section{SOME OPTIMIZATION LOGISTICS MODELS FOR TRANSPORTATION OF FRESH PALM OIL BUNCHES, COLOMBIA}

\author{
Wilson Adarme Jaimes \\ Ingeniero Industrial, M.Sc. en Ingeniería Logística. Esp. en Gerencia de la Producción, \\ Ph.D. (c) en Ingeniería Logística. Profesor Asistente Facultad de Ingeniería, \\ Universidad Nacional de Colombia, Sede Bogotá. \\ wadarmej@unal.edu.co \\ Carlos Fontanilla Díaz
}

Ingeniero de Producción Agroindustrial, M.Sc. en Ingeniería Industrial, CENIPALMA, Sección de Economía Agrícola, Centro de Investigación en Palma de Aceite cfontanilla@cenipalma.org

Martín Darío Arango Serna

Ingeniero Industrial. M.Sc. en Ingeniería de Sistemas. Especialista en Finanzas. Ph.D. en Ingeniería Industrial. Profesor Titular Facultad de Minas, Universidad Nacional de Colombia, Sede Medellín. mdarango@unal.edu.co

Fecha de recepción: 15 de febrero de 2011

Fecha de aprobación: 30 de junio de 2011

\section{RESUMEN}

Los modelos de optimización del transporte en el sector palmicultor, buscan disminuir el costo de operación logística asociado con la asignación de vehículos en las etapas de recolección y transporte de los Racimos de Fruto Fresco (RFF) aquí, se proponen dos modelos logísticos aplicables en la gestión del transporte en la agroindustria de la palma de aceite en Colombia. El primer modelo describe la operación general y garantiza el envío de la recolección desde los puntos de acopio interno hasta los puntos de acopio externo, y de estos últimos a la planta productora. Y el segundo propone la condición single sourcing (Única fuente), para asegurar que el fruto proveniente de un lote, se asigne a sólo un acopio externo y se faciliten acciones como la inspección de la producción y el control de calidad de los lotes. La importancia de plantear un modelo single sourcing genera distintas posibilidades para evaluar escenarios en donde se ven enfrentados a diario los agentes que intervienes en este sector, buscando mecanismos de coordinación e integración. El modelo single sourcing modifica las condiciones de operación y de esta manera, se constituye en una herramienta básica que permite medir incidencias técnicas y económicas de cambios en la forma como opera el sistema de transporte. Al final se presentan los resultados, conclusiones y recomendaciones de este trabajo.

Palabras clave: crossdock, single sourcing, logística de transporte, costos logisticos. 


\section{ABSTRACT}

The optimization transportation models in the oil palm industry aim to reduce costs by logistics operations related to vehicle allocation during collection and transportation phases of fresh fruit bunches (FFB). Here we propose two logistic models pertinent to transportation management in the agro-industry of palm oil in Colombia. The first one describes a general operation and guarantees the delivery of the entire collection from collection centers internal to external collection locations and from the latter to a manufacturing plant. The second one proposes the single sourcing to ensure that a fruit batch could be sent to only one external collection in order to facilitate actions, such as production inspection and quality control of batches. The importance of this model generates several possibilities to evaluate scenarios where they are faced daily by actors involved in this sector, seeking coordination and integration mechanisms. This model also changes the operating conditions and thus constitutes a basic tool to measure technical and economic implications of changes in the way a transportation system operates. Finally, we present findings, conclusions and recommendations of this work.

Key words: crossdock, single sourcing, transportation logistics, logistics costs.

\section{INTRODUCCIÓN}

En la actualidad la palma de aceite es el cultivo de mayor crecimiento en Colombia, abastece la mayor parte del mercado nacional de aceites y grasas, y ha mantenido una presencia importante dentro de los rubros de exportación. Las ventajas comparativas de este cultivo tropical en Colombia, sus tendencias de desarrollo y el dinamismo de los mercados nacionales e internacionales de grasas y aceites, así como los biocombustibles, determinan un potencial de crecimiento de esta oleaginosa muy favorable para el País [1].

Colombia es uno de los países con más tierras aptas para la siembra de palma, al contar con 3.5 millones de hectáreas sin alguna restricción para la plantación de este cultivo. De estas hectáreas, el $36 \%$ se encuentran en siete departamentos de la costa Caribe, donde se cuenta con una infraestructura de puertos marítimos para el comercio exterior [2].

La dinámica de la agroindustria de palma de aceite en Colombia, presenta rasgos relevantes en aspectos relacionados con sus procesos técnicos, operativos y de soporte. Esta investigación se orienta a configurar modelos matemáticos que soporten la gestión del transporte en este sector, y hacen referencia a cómo gestionar los costos de operación logística asociados al transporte donde confluyen 
medios, equipos, tecnologías y diversos sistemas utilizados para la manutención y desplazamiento de racimos de frutos frescos (RFF), desde la finca hasta la planta de beneficio.

El área sembrada en Colombia con palma de aceite, viene creciendo a una tasa del $10,5 \%$ anual, pasando de 157.327 hectáreas en 2000 a 316.402 en 2007 ; el número de plantas de beneficio de Aceite Crudo de Palma (ACP), pasó de 51 a 53, durante el mismo período [3]; en el año 2000, a una planta de beneficio le correspondía atender en promedio 3.000 hectáreas; en 2007, este número ascendió a 6.000 hectáreas. Lo anterior, además de sugerir un aumento en el factor de utilización de la capacidad instalada de las plantas de beneficio y su enfoque cada vez mayor hacia economías de escala, ha tenido variaciones sobre la distancia recorrida para transportar los RFF desde el campo hasta la planta de beneficio.

\section{ANTECEDENTES MODELOS DE TRANSPORTE - PALMA DE ACEITE}

En los costos de producción de Aceite Crudo de Palma (ACP) en Colombia, la cosecha, el transporte de RFF y la extracción del aceite, participan con el 16\%, 5\%, y $21 \%$, respectivamente [4]. Por lo menos el $5 \%$ del costo de producción de una tonelada de ACP corresponde al transporte del fruto; el tiempo que transcurre entre el corte del racimo y su llegada a la planta de beneficio es definitivo, y proporcional al deterioro de la calidad del aceite, ya que al tratarse de un producto perecedero, una vez cortados los RFF, se acelera su proceso de acidificación. La diferencia en el precio entre un aceite de buena o baja calidad es cercana al $5 \%$, lo cual implica pagos entre US 20 y US 25 por tonelada de aceite de palma [5]. Entre los medios de transporte que llegan a los puntos de acopio, se destacan los camiones de auto carga [6], ubicados en las fincas con una caja contenedora vacía que se intercambia por una llena de RFF, para transportarla y depositarla en la planta de beneficio; los camiones son cargados manualmente o por mecanismos hidráulicos de alce de mallas. El uso de tractores y zorras de tres o cinco toneladas [7] y/o el uso de cable vías [8] para el transporte de mallas cargadas con fruto desde el campo hasta la planta de beneficio, son una alternativa que se viene evaluando.

La investigación definió dos tipos de modelos con los cuales se determinan los costos de transporte por tonelada, dependiendo del sistema de gestión utilizado; estos modelos tienen la versatilidad para adaptarse al sector, de acuerdo con parámetros y circunstancias que la operación presente. De esta manera, se describe una evaluación de antecedentes, la metodología de la investigación, definición de variables, parámetros y la configuración de los modelos acompañada de ejemplos para determinar su consistencia y aplicabilidad, así como las conclusiones que se derivan del estudio. Para mejorar el sistema logístico de transporte de fruto desde las fincas hasta la planta de beneficio en la agroindustria de la palma de aceite en 
Colombia, se encuentran algunas investigaciones que determinan cómo operar y/o qué herramientas utilizar. Entre los referentes y estudios locales y mundiales relevantes encontrados sobre transporte de RFF, están los que proponen un modelo de localización para la producción de palma de aceite en Nigeria [9]. Existe otro relacionado con actividades logísticas en una plantación del Magdalena (Unipalma), zona palmera oriental de Colombia [4]. También un estudio de tiempos, movimientos y costeo de actividades de transporte de RFF en Colombia [10], y un modelo matemático (no lineal, mixto entero), para optimizar los eslabones de cosecha y de extracción de aceite en la cadena de palma [11].

Otros estudios proponen la necesidad de orientar la competitividad del sector palmero con la reducción de costos al optimizar la cadena de aceite de palma, con la utilización de modelos de programación lineal [12]. Existe un sistema integrado para soporte de decisiones, basado en algoritmos genéticos multi-objetivo y lógica difusa, para optimizar la cadena de abasto de la industria de biodiesel en Indonesia [13], y modelos de programación entera para resolver un problema de transporte de aceite crudo de palma en Malasia [14]. Se han desarrollado modelos matemáticos de programación lineal entera mixta para planificar el sector del biodiesel en Colombia [15], y un trabajo en una plantación de palma de aceite de 450 hectáreas, con un modelo de asignación de personal de cosecha a los lotes, basado en las competencias individuales de cada cosechero que logra incrementar los rendimientos de los operarios en un $6,4 \%$, con respecto de la asignación manual que se venía realizando [16].

Existe una revisión bibliográfica sobre el uso de modelos de optimización en la cadena de abastecimiento de palma de aceite en Colombia. Dentro de los hallazgos, se encuentran limitados estudios al respecto que proponen un modelo de programación entera mixta (PEM), con el cual ofrecen una reducción en los costos de transporte [17]. Con base en revisión de antecedentes, investigaciones sobre modelación y evaluación detallada de los modus operandi de la fincas palmicultoras en Colombia, se formulan los modelos matemáticos de programación entera mixta que incluyen definición de parámetros, variables y corridas en lenguajes de programación, con características de flexibilidad, lo cual permite incorporar nuevos parámetros y criterios para optimizar el transporte de RFF en las plantaciones de palma de aceite. A continuación, se presenta la metodología que siguió la definición de los modelos matemáticos propuestos.

\section{METODOLOGÍA}

La estructura del trabajo de investigación se puede ver en dos partes: primero, se describe el modelo general de distribución dentro de las formas de organización entre clientes y proveedores que ha desarrollado la literatura. Después, se analiza 
cuáles son sus rasgos básicos y las razones que han propiciado su acogimiento como tecnología organizativa. A continuación, se estudian sus beneficios en términos de reducción de precios del proceso, y se hace lo mismo con la metodología Single Sourcing, y se concluye, analizando la importancia del comportamiento de cada modelo y la conveniencia de utilizar cada uno, según el contexto en donde se utilice.

A partir de un acercamiento al modus operandi del sistema de transporte de RFF en diferentes plantíos de palma de aceite en Colombia, se identificaron parámetros, variables y restricciones, y con base en ellos, se configuraron los modelos respectivos, utilizando el software General Algebraic Modeling System (GAMS); se caracterizaron modos y medios de transporte, su capacidad, velocidad, tiempos de carga y descarga, sistemas de comunicación y costos, entre otros.

El proceso de transporte de RFF tiene varias etapas (figura 1): se inicia en los lotes de cosecha cuando la producción obtenida se deposita en el punto de acopio asociado con el mismo (llamado en este caso PUNTO INTERNO); (i): allí, un vehículo recoge los RFF y los transporta a un punto de acopio externo (Cross dock); (j): con una capacidad determinada; posteriormente, otro vehículo los transporta del punto de acopio externo (j), a las plantas de beneficio (t).

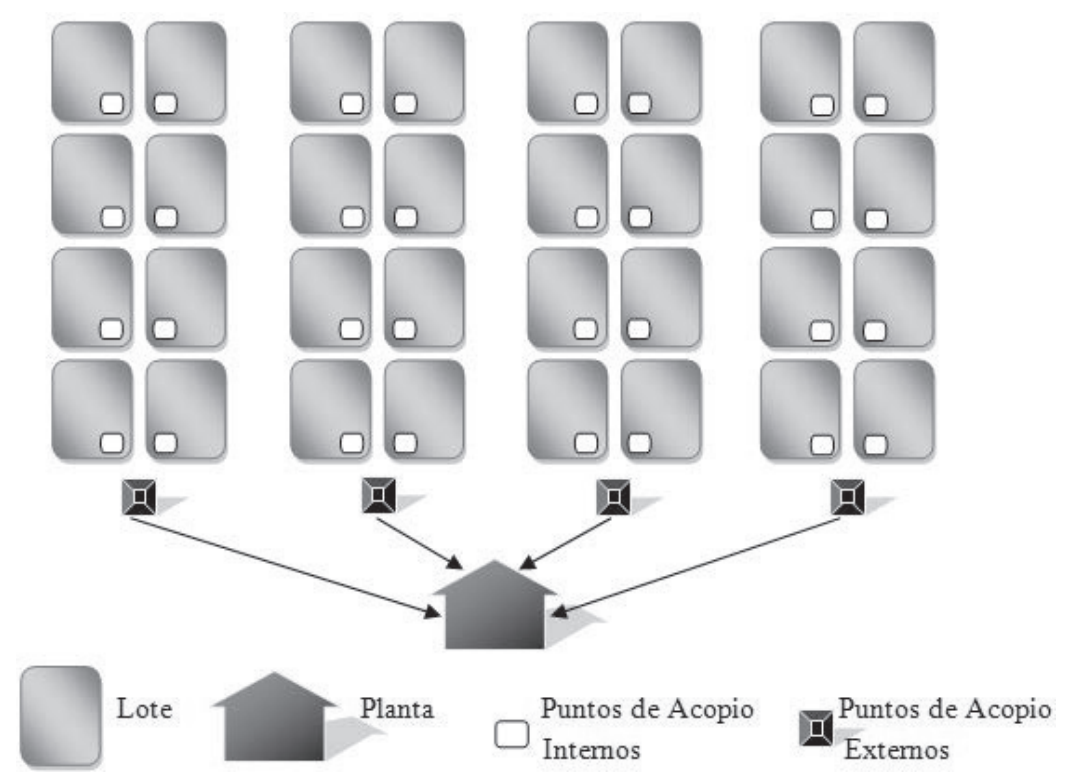

Figura 1. Proceso de transporte de Fruto de palma de aceite. Fuente: Fontanilla, et al. 2010 [7]

La metodología contempló evaluar de diferentes fincas productoras de palma de aceite, los rasgos y características de los sistemas de transporte, variedad de 
medios, distinguidos por capacidad de carga por viaje y/o rendimiento, número de viajes por período (día), tiempos de cargue y descargue, número de viajes máximo por período que puede realizar un camión, y el número de camiones disponibles de cada tipo, costos unitarios de transporte $(\$ / \mathrm{km})$, que pueden variar en función del medio y la distancia (la versatilidad de la propuesta puede permitir colocar costos diferenciadores por $\mathrm{km}$ y medio). Fue necesario conocer el costo de desplazamiento en $\$ / \mathrm{km}$ y el costo fijo por período de cada medio que por economías de escala, puede permitir mayor eficiencia, según el tamaño del camión cuando las distancias por recorrer son largas. Se establecieron las distancias en $\mathrm{km}$ desde cada punto interno (i) hasta cada punto externo (j), la velocidad por tipo de camión, la producción en toneladas esperada de cada finca agrupada en el punto interno (i). El programa de cosecha por período (diario), en cada punto interno (i), la capacidad de los puntos externos (j) y las plantas (t).

El Single Sourcing se adopta como la decisión voluntaria de una empresa por vincularse con un número muy limitado de proveedores. No existe Single Sourcing porque el origen sea único debido a un diseño peculiar, la localización u otros factores que provocan que exista un origen exclusivo, sino que se caracteriza porque se trata de una elección realizada sobre una base de posibles opciones. El Single Sourcing se establece en aras de una mayor eficiencia en la relación. Se trabaja conjuntamente para ofrecer un mayor valor agregado a la producción del socio, y para que este esfuerzo alcance su máxima efectividad, no se debe dispersar. El Single Sourcing nunca llega al extremo de existir un único proveedor para una pieza o un conjunto funcional, ya que al fabricante le interesa crear competencia entre sus proveedores, con el objeto de conseguir ventajas en relación con: precio, calidad, plazos de entrega o flexibilidad de la producción.Teniendo en cuenta lo anterior, Single Sourcing se define como un lote que sólo se puede enviar a un centro de acopio externo (j); sin embargo, un centro de acopio (j) puede recibir de más de un punto de recolección interna.

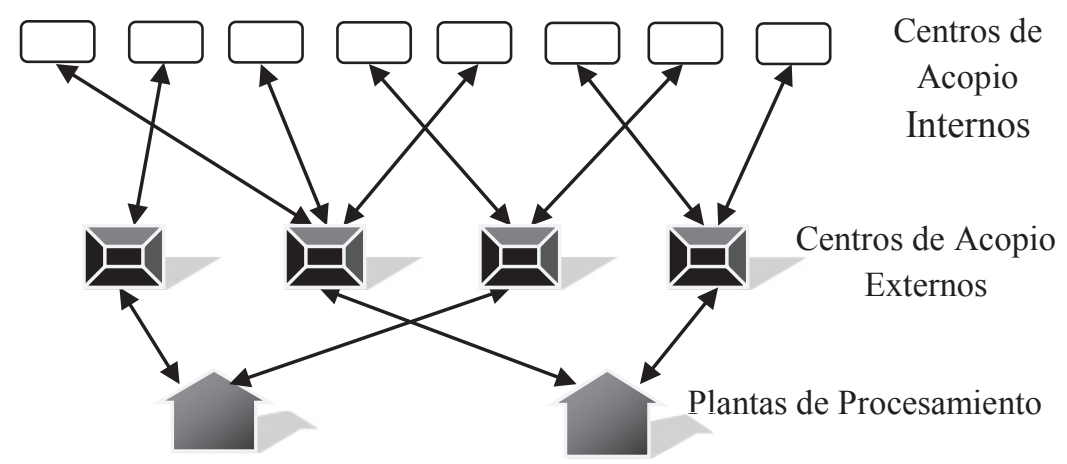

Figura 2. Situación con Single Sourcing. 
Por razones administrativas y técnicas, y aspectos fisicoquímicos, sólo en los puntos externos (j) se podrá combinar cargas provenientes de los puntos internos (i). La figura 2 ilustra este hecho, donde los centros de acopio interno no pueden hacer envíos a más de un centro de acopio externo; esto se configura en la situación Single Sourcing.

\section{FORMULACIÓN MODELO GENERAL PROPUESTO}

Con base en el contexto del transporte de RFF, se describe una situación típica y se propone un modelo que por sus características, tiene potencialidad en su adopción y en sus resultados operativos y económicos, como se demostrará, soportado en un análisis de parámetros y factores relevantes de las zonas geográficas atendidas. Este modelo tiene la versatilidad de servir como pivote y adecuarse a las particularidades que demanda la operación del transporte de RFF. La formulación genérica de esta situación es la siguiente:

Objetivo: Determinar un plan de acopio de RFF de palma de aceite (número de viajes y cantidad para transportar), que produzca el menor costo posible por tonelada.

\section{Función objetivo: $F=f(p l a n$ de distribución $)=$ función costo}

\section{Variables de decisión}

$\boldsymbol{x}_{i j k}$ : Cantidad de ton., para transportar desde el origen (punto de acopio interno), "i" al destino (punto de acopio externo), "j" en el medio de transporte (camión), "k" donde $i=1,2, \ldots m ; j=1,2, \ldots, n \quad y k=1,2, \ldots, r$

$\boldsymbol{Q}_{i j k}$ : Cantidad de viajes por programar desde el origen "i" al destino"j" en el medio de transporte (camión), "k" donde $i=1,2, \ldots m ; j=1,2, \ldots, n$ y $k=1,2, \ldots, r$

$\boldsymbol{Y}_{j t l}$ : Cantidad de toneladas para transportar desde el origen "j" al destino (planta), "t" en el medio de transporte (camión), "I" donde $\mathrm{j}=1,2, \ldots n ; t=1,2, \ldots, s \quad$ y $l=1,2, \ldots, p$ $\boldsymbol{P}_{j t l}$ : Cantidad viajes por programar desde el origen "j" al destino (planta), "t" en el medio de transporte (camión), "l" donde $\mathrm{j}=1,2, \ldots n ; t=1,2, \ldots, s \quad$ y $l=1,2, \ldots, p$

$\boldsymbol{B}_{\boldsymbol{k}}$ : Variable binaria que activa el costo fijo del medio de transporte (camión), "k", en el caso de que este medio realice por lo menos un viaje. $k=1,2, \ldots, r$

$\boldsymbol{B}_{l}$ : Variable binaria que activa el costo fijo del medio de transporte (camión), "l", en el caso de que este medio realice por lo menos un viaje. $l=1,2, \ldots, p$ 


\section{Parámetros}

$C_{i j k}$ : Costo de un viaje en pesos desde el origen “i” al destino “j”, usando el medio de transporte (camión), "k" donde $i=1,2, \ldots m ; j=1,2, \ldots, n$ y $k=1,2, \ldots, r$

$\boldsymbol{S}_{j t l}$ : Costo de un viaje en pesos desde el origen "j" al destino (planta) "t" en el medio de transporte (camión) "l" donde $j=1,2, \ldots, n ; t=1,2, \ldots, s$ y $l=1,2, \ldots, p$

$\boldsymbol{C U}_{i j k}$ : Costo por kilómetro en pesos desde el origen "i" al destino "j", usando el medio de transporte (camión), "k" donde $i=1,2, \ldots m ; j=1,2, \ldots, n$ y $k=1,2, \ldots, r$ $\boldsymbol{C} \boldsymbol{W}_{\boldsymbol{j} t \boldsymbol{t}}$ : Costo por kilómetro pesos desde el origen "j" al destino (planta), " $\mathrm{t}$ " en el medio de transporte (camión), "l" donde $j=1,2, \ldots, n ; t=1,2, \ldots, s \quad$ y $l=1,2, \ldots, p$ $\boldsymbol{C F}_{\boldsymbol{k}}$ : Costo fijo diario de operación del medio de transporte "k" donde $k=1,2, \ldots r$ $\boldsymbol{C F}_{\boldsymbol{l}}$ : Costo fijo diario de operación del medio de transporte "I" donde $l=1,2, \ldots p$ $\boldsymbol{P R \boldsymbol { O } _ { i }}$ : Cantidad en toneladas que se cosecha en las fincas asociadas con el punto de acopio interno "i" donde $i=1,2, \ldots m$

$\boldsymbol{C A}_{\boldsymbol{j}}$ : Cantidad máxima en toneladas que puede ser almacenada en el punto de acopio externo "j" donde $j=1,2, \ldots n$

$\boldsymbol{C} \boldsymbol{P}_{\boldsymbol{t}}$ : Cantidad máx., en ton., que puede ser procesada en la planta "t"; $t=1,2, \ldots s$

$\boldsymbol{N} \boldsymbol{V}_{\boldsymbol{k}}$ : Cantidad máxima de viajes al día que puede hacer un medio de transporte (camión), "k" donde $k=1,2, \ldots r$

$\boldsymbol{N} \boldsymbol{V}_{l}$ : Cantidad máxima de viajes al día que puede hacer un medio de transporte (camión), "l" donde $l=1,2, \ldots p$

$\boldsymbol{K} \boldsymbol{D}_{\boldsymbol{k}}$ : Número disponible de camiones tipo " $\mathrm{k}$ " donde $k=1,2, \ldots r$

$\boldsymbol{K} \boldsymbol{D}_{l}$ : Número disponible de camiones tipo "l" donde $l=1,2, \ldots p$

$\boldsymbol{D} \boldsymbol{I}_{i j}$ : Distancia en km desde el origen "i" al destino "j"; $i=1,2, \ldots m$ y $j=1,2, \ldots, n$

$\boldsymbol{D} \boldsymbol{E}_{\boldsymbol{j} \boldsymbol{t}}$ : Distancia en km desde el origen "j" al destino "t"; j=1,2, ..n y $t=1,2, \ldots, s$

$\boldsymbol{C} C_{\boldsymbol{k}}$ : Capacidad máxima de carga en toneladas por viaje del medio de transporte (camión), "k" donde $k=1,2, \ldots r$

$C C_{l}$ : Capacidad máxima de carga en toneladas por viaje del medio de transporte (camión) "l" donde $l=1,2, \ldots p$

$\boldsymbol{T C} \boldsymbol{D}_{\boldsymbol{k}}$ : Tiempo de cargue y descargue (en horas), de los RFF en el medio de transporte (camión), " $\mathrm{k}$ " donde $k=1,2, \ldots r$

$T \boldsymbol{C}_{\boldsymbol{l}}$ : Tiempo de cargue (en horas), de los RFF en el medio de transporte "l" donde $l=1,2, \ldots p$

$T D_{l}$ : Tiempo de descargue (en horas), de los RFF en medio de transporte "I" donde $l=1,2, \ldots p$

$\boldsymbol{T} \boldsymbol{M}_{\boldsymbol{k}}$ : Tiempo máximo en horas de trabajo por día del medio de transporte (camión), "k" donde $k=1,2, \ldots r$

$\boldsymbol{T} \boldsymbol{M}_{l}$ : Tiempo máximo en horas de trabajo por día del medio de transporte (camión), "l" donde $l=1,2, \ldots p$

$V I_{i j k}$ : Velocidad de recorrido ( $\mathrm{km} / \mathrm{hora}$ ), del medio de transporte (camión), "k" desde el origen "i" al destino "j" donde $\mathrm{k}=1,2, \ldots r ; i=1,2, \ldots, m ; j=1,2, \ldots n$ 
$\boldsymbol{V} \boldsymbol{E}_{\boldsymbol{j} t l}$ : Velocidad de recorrido ( $\mathrm{km} / \mathrm{hora}$ ), del medio de transporte (camión), "l" desde el origen "j" al destino "t" donde $\mathrm{I}=1,2, \ldots p ; j=1,2, \ldots, n ; t=1,2, \ldots \mathrm{S}$

$M$ : Número Grande

Función Objetivo: Cada familia de restricciones garantiza el cumplimiento de una condición del sistema, así: La expresión (1) es la función objetivo para minimizar el costo total por ton., para transportar. Dentro de los costos están los asociados al viaje de cada camión, dependiendo del costo por kilómetro y la distancia recorrida; y el costo fijo en el cual se incurre por utilizar un vehículo.

$\min Z$

$=\frac{\sum_{i=1}^{m} \sum_{j=1}^{n} \sum_{k=1}^{r} C_{i j k} Q_{i j k}+\sum_{j=1}^{n} \sum_{t=1}^{S} \sum_{l=1}^{p} S_{j t l} P_{j t l}+\sum_{k=1}^{r} C F_{k} * B_{k}+\sum_{l=1}^{p} C F_{l} * B_{l}}{\sum_{i=1}^{m} P R O_{i}}$

La restricción (2) garantiza que la cantidad recolectada en cada centro de acopio interno, sea igual a la cantidad que se distribuye en los distintos medios a los centros de acopio externos.

s.a.

$$
\sum_{k=1}^{r} \sum_{j=1}^{n} X_{i j k}=P R O_{i} \quad \forall i=1,2, \ldots, m
$$

La restricción (3) es una ecuación de balance de flujo (entra - sale), en centro de acopio externo.

$$
\sum_{k=1}^{r} \sum_{i=1}^{m} X_{i j k}=\sum_{l=1}^{p} \sum_{t=1}^{s} Y_{j t l} \quad \forall j=1,2, \ldots, n
$$

La restricción (4) relaciona la cantidad por transportar entre un origen "i" y un destino "j" con la capacidad del medio de transporte "k" para determinar cantidad de viajes por realizar entre las dos locaciones.

$$
X_{i j k} \leq Q_{i j k} * C C_{k} \quad \forall i=1,2, \ldots, m ; j=1,2, \ldots, n y k=1,2, \ldots, r
$$

La restricción (5) relaciona la cantidad por transportar entre un origen "j" y un destino "t" con la capacidad del medio de transporte "l" para determinar la cantidad de viajes por realizar entre las dos locaciones.

$$
Y_{j t l} \leq P_{j t l} * C C_{l} \quad \forall j=1,2, \ldots, n ; t=1,2, \ldots, \text { s } y l=1,2, \ldots, p
$$


La restricción (6) contabiliza la cantidad de viajes que hará el medio de transporte tipo "k" para determinar si se incurre o no, en el costo fijo de operación.

$$
\sum_{i=1}^{m} \sum_{j=1}^{n} Q_{i j k} \leq B_{k} * M \quad \forall k=1,2, \ldots, r
$$

La restricción (7) contabiliza la cantidad de viajes que hará el medio de transporte tipo "I", para determinar si se incurre o no, en el costo fijo de operación.

$$
\sum_{j=1}^{n} \sum_{t=1}^{s} P_{j t l} \leq B_{l} * M \quad \forall l=1,2, \ldots, p
$$

La restricción (8) garantiza que la cantidad que llega a un centro de acopio externo "j", no exceda su capacidad de almacenamiento.

$$
\sum_{k=1}^{r} \sum_{i=1}^{m} X_{i j k} \leq C A_{j} \forall j=1,2, \ldots, n
$$

La restricción (9) garantiza que la cantidad que llega a la planta "t", no exceda su capacidad de proceso.

$$
\sum_{l=1}^{p} \sum_{j=1}^{n} Y_{j t l} \leq C P_{t} \forall t=1,2, \ldots, s
$$

La restricción (10), afecta el número de viajes por realizar en el medio "k", de acuerdo con la cantidad máx. de viajes por un camión "k", y el número disponible de medios de ese tipo.

$$
\sum_{i=1}^{m} \sum_{j=1}^{n} Q_{i j k} \leq N V_{k} * K D_{k} \forall k=1,2, \ldots, r
$$

La restricción (11), afecta el número de viajes por realizar en el medio "I", de acuerdo con la cantidad máx. de viajes por un camión "l", y el número disponible de camiones de ese tipo.

$$
\sum_{j=1}^{n} \sum_{t=1}^{s} P_{j t l} \leq N V_{l} * K D_{l} \forall l=1,2, \ldots, p
$$


La restricción (12), afecta el tiempo diario de operación del medio tipo "k", de acuerdo con el tiempo máximo por día y el número de medios de ese tipo que están disponibles.

$$
\sum_{i=1}^{m} \sum_{j=1}^{n}\left(\frac{D I_{i j}}{V I_{i j k}}+T C D_{k}\right) Q_{i j k} \leq T M_{k} * K D_{k} \forall k=1,2, \ldots, r
$$

La restricción (13), afecta el tiempo diario de operación del medio tipo "l", de acuerdo con el tiempo máximo por día y el número de medios de ese tipo que están disponibles.

$$
\sum_{j=1}^{n} \sum_{t=1}^{s}\left(\frac{D E_{j t}}{V E_{j t l}}+T C_{l}+T D_{l}\right) P_{j t l} \leq T M_{l} * K D_{l} \forall l=1,2, \ldots, p
$$

La expresión (14), desarrolla el cálculo del costo asociado a un viaje entre un origen "i" y un destino "j" con el medio "k", teniendo en cuenta que el recorrido del vehículo descargado cuesta por ejemplo, el 50\% del valor cuando está cargado.

$$
C_{i j k}=1,5 * C U_{i j k} * D I_{i j} i=1,2, \ldots, m ; j=1,2, \ldots, n y k=1,2, \ldots, r
$$

La expresión (15), desarrolla el cálculo del costo asociado a un viaje entre un origen "j" y un destino "t" con el medio "l", teniendo en cuenta que el recorrido del vehículo descargado cuesta por ejemplo, el 50\% del valor cuando está cargado.

$$
S_{j t l}=1,5 * C W_{j t l} * D E_{j t} j=1,2, \ldots, n ; t=1,2, \ldots, \text { s y } l=1,2, \ldots, p
$$

La restricción (16), garantiza la no negatividad de las variables y la naturaleza de cada una.

$$
X_{i j k}, Y_{j t l} \geq 0 ; \quad Q_{i j k}, P_{j t l} \text { enteras }
$$

\subsection{EJEMPLO: SITUACIÓN GENERAL}

Dadas las restricciones de recursos de los investigadores y de información en las fincas, se consideraron seis puntos de acopio interno, dos tipos de vehículo de transporte interno, tres puntos de acopio externos y dos tipos de vehículos de transporte a una planta de beneficio. La figura 3 representa esta situación, común en las zonas palmicultoras. 


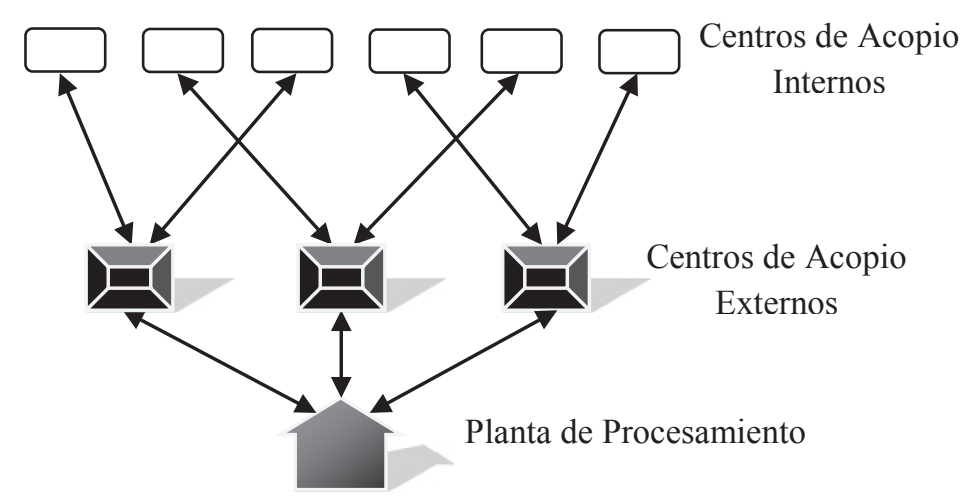

Figura 3. Esquema de la situación para resolver

Con base en la operación de las fincas y los registros en los centros de acopio, se han construido las matrices de producción de los lotes y los costos de transporte. La producción por recoger en cada lote (tabla 1), para el período de evaluación fue:

Tabla 1. Producción de los lotes, para el ciclo de cosecha en estudio

\begin{tabular}{|c|c|}
\hline Lote (Acopio interno i) & Producción (ton-día) \\
\hline $\mathbf{1}$ & 7 \\
\hline 2 & 3 \\
\hline 3 & 14 \\
\hline 4 & 2 \\
\hline 5 & 2 \\
\hline 6 & 7 \\
\hline
\end{tabular}

Los costos de la tabla 2, se dan para el caso del vehículo cargado. Cuando el vehículo regresa descargado, se asume el $50 \%$ del mismo (en las fincas los camiones transportan en contrasentido efluentes como fibras para depositar en los lotes, todas estas situaciones el modelo permite incluirlas).

Tabla 2. Costo por kilómetro de acuerdo con cada trayecto $(\$ / \mathrm{km})$

\begin{tabular}{|c|c|c|c|}
\hline \multirow{2}{*}{ Acopios internos i } & \multicolumn{3}{|c|}{ Acopios externos j (pesos colombianos COP) } \\
\cline { 2 - 4 } & $\mathbf{1}$ & $\mathbf{2}$ & $\mathbf{3}$ \\
\hline $\mathbf{1}$ & 1.317 & 3.244 & 1.790 \\
\hline $\mathbf{2}$ & 1.790 & 3.244 & 1.317 \\
\hline $\mathbf{3}$ & 1.317 & 3.244 & 1.317 \\
\hline $\mathbf{4}$ & 1.317 & 1.790 & 3.244 \\
\hline $\mathbf{5}$ & 1.317 & 1.317 & 1.790 \\
\hline $\mathbf{6}$ & 1.317 & 1.317 & 1.317 \\
\hline
\end{tabular}


Tabla 3. Distancia entre los puntos de acopio internos y externos (km)

\begin{tabular}{|c|c|c|c|}
\hline Acopios internos i & \multicolumn{3}{|c|}{ Acopios externos j } \\
\cline { 2 - 4 } & $\mathbf{1}$ & $\mathbf{2}$ & $\mathbf{3}$ \\
\hline $\mathbf{1}$ & 16 & 20 & 14 \\
\hline $\mathbf{2}$ & 5 & 19 & 10 \\
\hline $\mathbf{3}$ & 14 & 8 & 3 \\
\hline $\mathbf{4}$ & 5 & 11 & 6 \\
\hline $\mathbf{5}$ & 4 & 10 & 3 \\
\hline $\mathbf{6}$ & 14 & 15 & \\
\hline
\end{tabular}

Se estimaron las velocidades medias entre los puntos de acopio internos y externos, que se presentan en la tabla 4.

Tabla 4. Velocidades de los vehículos entre los acopios internos y externos $(\mathrm{km} / \mathrm{h})$

\begin{tabular}{|c|c|c|c|}
\hline \multirow{2}{*}{$\begin{array}{c}\text { Acopios } \\
\text { internos } \mathbf{i}\end{array}$} & \multicolumn{3}{|c|}{ Acopios externos j } \\
\cline { 2 - 4 } & $\mathbf{1}$ & $\mathbf{2}$ & $\mathbf{3}$ \\
\hline $\mathbf{1}$ & 30 & 20 & 25 \\
\hline $\mathbf{2}$ & 25 & 20 & 30 \\
\hline $\mathbf{3}$ & 30 & 20 & 30 \\
\hline $\mathbf{4}$ & 30 & 25 & 20 \\
\hline $\mathbf{5}$ & 30 & 30 & 25 \\
\hline $\mathbf{6}$ & 30 & 30 & 30 \\
\hline
\end{tabular}

Las velocidades anteriores son el promedio con carga o sin carga, entre puntos de acopio externos y planta de beneficio; el costo de transporte por kilómetro se estimó en \$1.317 COP, y con las distancias, se calculó el costo de transporte externo (tabla 5).

Tabla 5. Costo de transporte entre los puntos de acopio externo y la planta de beneficio (\$).

\begin{tabular}{|c|c|c|}
\hline $\begin{array}{c}\text { Punto de acopio } \\
\text { externo j }\end{array}$ & Distancia & $\begin{array}{c}\text { Costo de transporte } \\
\text { (Pesos Colombianos COP) }\end{array}$ \\
\hline $\mathbf{1}$ & 8 & 10.533 \\
\hline $\mathbf{2}$ & 12 & 15.800 \\
\hline $\mathbf{3}$ & 8 & 10.533 \\
\hline
\end{tabular}

Se dispone de dos vehículos para el transporte interno, uno de 10 ton y otro de 5 ton y dos vehículos para el transporte externo con capacidad de 10 y 12 toneladas. El máximo número de viajes por día, para los diferentes vehículos, se estabelció en cuatro. El costo de la carga está asociado a las toneladas transportadas y existe un costo fijo por día, que ha sido estimado para el vehículo de 5 toneladas, en $\$ 4.000$; para el de 10 toneladas, $\$ 5.000$ y para el de 12 toneladas, $\$ 6.000$. 
La capacidad de la planta es de 500 ton/día y la capacidad de cada centro de acopio externo es de 15 toneladas. Los tiempos de cargue y descargue de RFF en vehículo "k" en horas, se calculó en 0,30 y el tiempo de cargue de RFF en vehículo "l", se determinó en 0,1 horas y el tiempo de descargue para este tipo de vehículo, se calculó en 0,5 horas. El tiempo de trabajo para los vehiculos tipo "k" o "l" es de 8 horas/día.

\subsection{MODELO GENERAL CON SINGLE SOURCING}

El contexto estudiado establece una condición de carácter administrativo, relacionada con la necesidad de garantizar que toda la recolección de un centro de acopio interno " $i$ ", sólo puede ser conducida a un único centro de acopio externo "j". Esta circunstancia es conocida como single sourcing y es necesaria para facilitar el proceso de control de calidad, seguimiento de registros de envíos entre origen y destino, gestión de transportistas y proveedores; estas decisiones en este sector, tienen implicaciones económicas y técnicas y en tal sentido, se formula esta situación como aporte para el sector. La formulación de la situación, teniendo en cuenta el single sourcing es la siguiente:

\section{Variables de decisión}

$\boldsymbol{x}_{i j k}$ : Cantidad de toneladas por transportar desde el origen (punto de acopio interno), "i" al destino (punto de acopio externo), "j" en el medio de transporte (camión), "k" donde $i=1,2, \ldots m ; j=1,2, \ldots, n \quad y k=1,2, \ldots, r$

$\boldsymbol{Q}_{i j k}$ : Cantidad de viajes por programar desde el origen "i" al destino "j", en el medio de transporte (camión), "k" donde $i=1,2, \ldots m ; j=1,2, \ldots, n \quad y k=1,2, \ldots, r$

$\boldsymbol{Y}_{j t l}$ : Cantidad de toneladas por transportar desde el origen "j" al destino (planta), "t" en el medio de transporte (camión), "l" donde $\mathrm{j}=1,2, \ldots n ; t=1,2, \ldots, s \quad$ y $l=1,2, \ldots, p$ $\boldsymbol{P}_{j t l}$ : Cantidad a viajes por programar desde el origen "j" al destino (planta), "t" en el medio de transporte (camión), "l" donde $\mathrm{j}=1,2, \ldots n ; t=1,2, \ldots, s \quad y l=1,2, \ldots, p$

$\boldsymbol{F}_{\boldsymbol{i j}}$ : Variable binaria que indica si el origen "i" envía o no, su recolección al destino "j" donde $i=1,2, \ldots m ; j=1,2, \ldots, n$

$\boldsymbol{B}_{\boldsymbol{k}}$ : Variable binaria que activa el costo fijo del medio de transporte (camión), "k", en el caso de que este medio realice por lo menos un viaje. $k=1,2, \ldots, r$

$\boldsymbol{B}_{\boldsymbol{l}}$ : Variable binaria que activa el costo fijo del medio de transporte (camión), "l", en el caso de que este medio realice por lo menos un viaje. $l=1,2, \ldots, p$

\section{Parámetros}

$C_{i j k}$ : Costo de un viaje en pesos, desde el origen "i" al destino "j", usando el medio de transporte (camión), "k" donde $i=1,2, \ldots m ; j=1,2, \ldots, n$ y $k=1,2, \ldots, r$ 
$\boldsymbol{S}_{j t l}$ : Costo de un viaje en pesos, desde el origen "j" al destino (planta), "t" en el medio de transporte (camión), "l" donde $j=1,2, \ldots, n ; t=1,2, \ldots, s$ y $l=1,2, \ldots, p$

$\boldsymbol{C} \boldsymbol{U}_{i j k}$ : Costo por kilómetro en pesos, desde el origen "i" al destino "j", usando el medio de transporte (camión), "k" donde $i=1,2, \ldots m ; j=1,2, \ldots, n$ y $k=1,2, \ldots, r$ $C \boldsymbol{W}_{j t l}$ : Costo por kilómetro en pesos, desde el origen "j" al destino (planta), "t" en el medio de transporte (camión), "l" donde $j=1,2, \ldots, n ; t=1,2, \ldots, s$ y $l=1,2, \ldots, p$

$\boldsymbol{C F}_{\boldsymbol{k}}$ : Costo fijo diario de operación del medio de transporte "k" donde $k=1,2, \ldots r$

$\boldsymbol{C F}_{\boldsymbol{l}}$ : Costo fijo diario de operación del medio de transporte "l" donde $l=1,2, \ldots p$

$\boldsymbol{P R O}_{i}$ : Cantidad en toneladas que se cosecha en las fincas asociadas al punto de acopio interno "i", donde $i=1,2, \ldots m$

$C \boldsymbol{A}_{\boldsymbol{j}}$ : Cantidad máxima en toneladas que puede ser almacenada en el punto de acopio externo "j", donde $j=1,2, \ldots n$

$\boldsymbol{C P}_{\boldsymbol{t}}$ : Cantidad máxima en toneladas que puede ser procesada en la planta " $\mathrm{t}$ ", donde $t=1,2, \ldots s$

$N V_{k}$ : Cantidad máxima de viajes al día que puede hacer un medio de transporte (camión), "k" donde $k=1,2, \ldots r$

$N V_{l}$ : Cantidad máxima de viajes al día que puede hacer un medio de transporte (camión), "l" donde $l=1,2, \ldots p$

$\boldsymbol{K} \boldsymbol{D}_{\boldsymbol{k}}$ : Número disponible de camiones tipo "k" donde $k=1,2, \ldots r$

$\boldsymbol{K} \boldsymbol{D}_{l}$ : Número disponible de camiones tipo "l" donde $l=1,2, \ldots p$

$\boldsymbol{D I}_{i j}$ : Distancia en kilómetros desde el origen (punto de acopio interno), "i" al destino (punto de acopio externo), "j" donde $i=1,2, \ldots m$ y $j=1,2, \ldots, n$

$\boldsymbol{D} \boldsymbol{E}_{\boldsymbol{j} \boldsymbol{t}}$ : Distancia en kilómetros desde el origen (punto de acopio externo), "j" al destino (planta), "t" donde $\mathrm{j}=1,2, \ldots n$ y $t=1,2, \ldots, s$

$\boldsymbol{C} C_{\boldsymbol{k}}$ : Capacidad máxima de carga en toneladas por viaje, del medio de transporte (camión), "k" donde $k=1,2, \ldots r$

$C C_{l}$ : Capacidad máxima de carga en toneladas por viaje, del medio de transporte (camión), "l" donde $l=1,2, \ldots p$

$\boldsymbol{T C} \boldsymbol{D}_{\boldsymbol{k}}$ : Tiempo de cargue y descargue (en horas), de los RFF en el medio de transporte (camión), " $\mathrm{k}$ " donde $k=1,2, \ldots r$

$\boldsymbol{T C}_{\boldsymbol{l}}$ : Tiempo de cargue (en horas), de los RFF en el medio de transporte (camión), "l" donde $l=1,2, \ldots p$

$T D_{l}$ :Tiempo de descargue (en horas), de los RFF en medio de transporte (camión), "l" $l=1,2, \ldots p$

$\boldsymbol{T} \boldsymbol{M}_{\boldsymbol{k}}$ : Tiempo máximo en horas de trabajo por día del medio del transporte (camión), "k" donde $k=1,2, \ldots r$

$\mathbf{T} \boldsymbol{M}_{l}$ : Tiempo máximo en horas de trabajo por día del medio del transporte (camión), "I" donde $l=1,2, \ldots p$

$\boldsymbol{V I}_{\boldsymbol{i j k}}$ : Velocidad de recorrido (en kilómetros por hora), del medio de transporte (camión), "k" desde el origen (punto de acopio interno), "i" al destino (punto de acopio externo), "j" donde $i=1,2, \ldots m ; j=1,2, \ldots, n y k=1,2, \ldots r$ 
$\boldsymbol{V} \boldsymbol{E}_{\boldsymbol{j} \boldsymbol{t l}}$ : Velocidad de recorrido (en kilómetros por hora), del medio de transporte (camión), "l" desde el origen (punto de acopio externo), "j" al destino (planta), "t" donde $j=1,2, \ldots n ; t=1,2, \ldots$, s y $l=1,2, \ldots p$

M: Número Grande

Cada familia de restricciones, garantiza el cumplimiento de una condición del sistema, así:

La expresión (17), función objetivo para minimizar el costo total por tonelada por distribuir. Dentro de los costos, están los asociados al viaje de cada camión, dependiendo del costo por kilómetro y la distancia recorrida; y el costo fijo en el cual se incurre por la utilización de un medio.

$\min Z$

$=\frac{\sum_{i=1}^{m} \sum_{j=1}^{n} \sum_{k=1}^{r} C_{i j k} Q_{i j k}+\sum_{j=1}^{n} \sum_{t=1}^{S} \sum_{l=1}^{p} S_{j t l} P_{j t l}+\sum_{k=1}^{r} C F_{k} * B_{k}+\sum_{l=1}^{p} C F_{l} * B_{l}}{\sum_{j=1}^{n} P R O_{i}}$

La restricción (18), garantiza que la cantidad recolectada en cada centro de acopio interno, sea igual a la cantidad que se distribuye en los distintos medios a los centros de acopio externos.

s.a.

$$
\sum_{k=1}^{r} \sum_{j=1}^{n} X_{i j k}=P R O_{i} \quad \forall i=1,2, \ldots, m
$$

La restricción (19), es una ecuación de balance de flujos (entra-sale), en el centro de acopio externo.

$$
\sum_{k=1}^{r} \sum_{i=1}^{m} X_{i j k}=\sum_{l=1}^{p} \sum_{t=1}^{s} Y_{j t l} \quad \forall j=1,2, \ldots, n
$$

La restricción (20), relaciona los envíos del origen "i" al destino "j" en los medios "k", con una variable binaria que indica la existencia $\left(F_{i j}=1\right)$, o no $\left(F_{i j}=0\right)$, de flujo entre los dos nodos.

$$
\sum_{k=1}^{p} X_{i j k} \leq F_{i j} * P R O_{i} \forall i=1, \ldots, m \text { y } j=1, \ldots, n
$$


La restricción (21), garantiza que todos los posibles envíos que salen del origen "i", sólo se dirijan a un único destino "j”, condición single sourcing.

$$
\sum_{j=1}^{n} F_{i j}=1 \quad \forall i=1, \ldots, m
$$

La restricción (22), relaciona la cantidad por transportar entre un origen "i" y un destino "j", con la capacidad del medio de transporte "k" para determinar la cantidad de viajes por realizar entre las dos locaciones.

$$
X_{i j k} \leq Q_{i j k} * C C_{k} \quad \forall i=1,2, \ldots, m ; j=1,2, \ldots, n y k=1,2, \ldots, r
$$

La restricción (23), relaciona la cantidad por transportar entre un origen "j" y un destino "t", con la capacidad del medio de transporte "l" para determinar la cantidad de viajes por realizar entre las dos locaciones.

$$
Y_{j t l} \leq P_{j t l} * C C_{l} \quad \forall j=1,2, \ldots, n ; t=1,2, \ldots, \text { s } y l=1,2, \ldots, p
$$

La restricción (24), contabiliza la cantidad de viajes que hará el medio de transporte tipo "k" para determinar si se incurre o no, en el costo fijo de operación.

$$
\sum_{i=1}^{m} \sum_{j=1}^{n} Q_{i j k} \leq B_{k} * M \quad \forall k=1,2, \ldots, r
$$

La restricción (25), contabiliza la cantidad de viajes que hará el medio de transporte tipo "l" para determinar si se incurre o no, en el costo fijo de operación.

$$
\sum_{j=1}^{n} \sum_{t=1}^{s} P_{j t l} \leq B_{l} * M \forall l=1,2, \ldots, p
$$

La restricción (26), garantiza que la cantidad que llega a un centro de acopio externo "j", no exceda su capacidad de almacenamiento.

$$
\sum_{k=1}^{r} \sum_{i=1}^{m} X_{i j k} \leq C A_{j} \forall j=1,2, \ldots, n
$$

La restricción (27), garantiza que la cantidad que llega a la planta "t", no exceda su capacidad de procesamiento. 


$$
\sum_{l=1}^{p} \sum_{j=1}^{n} Y_{j t l} \leq C P_{t} \forall t=1,2, \ldots, s
$$

La restricción (28), afecta los viajes destinados a hacer en el medio "k", de acuerdo con la cantidad máx. de viajes por un camión " $k$ " y el número disponible de camiones de ese tipo.

$$
\sum_{i=1}^{m} \sum_{j=1}^{n} Q_{i j k} \leq N V_{k} * K D_{k} \forall k=1,2, \ldots, r
$$

La restricción (29), afecta los viajes destinados a hacer en el medio "l", de acuerdo con la cantidad máx. de viajes por un camión "l" y el número disponible de camiones de ese tipo.

$$
\sum_{j=1}^{n} \sum_{t=1}^{s} P_{j t l} \leq N V_{l} * K D_{l} \forall l=1,2, \ldots, p
$$

La restricción (30), afecta el tiempo diario de operación del medio tipo "k", de acuerdo con el tiempo máximo por día y el número de medios de ese tipo que están disponibles.

$$
\sum_{i=1}^{m} \sum_{j=1}^{n}\left(\frac{D I_{i j}}{V I_{i j k}}+T C D_{k}\right) Q_{i j k} \leq T M_{k} * K D_{k} \forall k=1,2, \ldots, r
$$

La restricción (31), afecta el tiempo diario de operación del medio tipo "l", de acuerdo con el tiempo máximo por día y el número de medios de ese tipo que están disponibles.

$$
\sum_{j=1}^{n} \sum_{t=1}^{s}\left(\frac{D E_{j t}}{V E_{j t l}}+T C_{l}+T D_{l}\right) P_{j t l} \leq T M_{l} * K D_{l} \forall l=1,2, \ldots, p
$$

La expresión (32), desarrolla el cálculo del costo asociado a un viaje entre un origen "i" y un destino "j" con el medio "k", teniendo en cuenta que el recorrido del vehículo descargado cuesta el $50 \%$ del valor cuando está cargado.

$$
C_{i j k}=1,5 * C U_{i j k} * D I_{i j} i=1,2, \ldots, m ; j=1,2, \ldots, n y k=1,2, \ldots, r
$$


La expresión (33), desarrolla el cálculo del costo asociado a un viaje entre un origen "j" y un destino "t" con el medio "l", teniendo en cuenta que el recorrido del vehículo descargado cuesta el $50 \%$ del valor cuando está cargado.

$$
S_{j t l}=1,5 * C W_{j t l} * D E_{j t} j=1,2, \ldots, n ; t=1,2, \ldots, \text { s y } l=1,2, \ldots, p
$$

Las restricciones (34), garantizan la no negatividad de las variables y la naturaleza de cada una.

$$
X_{i j k}, Y_{j t l} \geq 0 ; \quad Q_{i j k}, P_{j t l} \text { enteras; } F_{i j} \text { binaria }
$$

\section{ANALISIS Y DISCUSION DE RESULTADOS}

Modelo General. El costo total de transporte por tonelada, de acuerdo con el modelo es de $\$ 5.706$ unidades monetarias, con lo cual se garantiza el envío de la totalidad de la recolección desde los puntos de acopio interno hasta los puntos de acopio externo, y de éstos últimos a una planta productora. Las cantidades de envío de la recolección de los distintos puntos de acopio interno a los puntos externos y de ellos a la planta, se asignaron como se aprecia en la figura 4.

Las restricciones de balance y capacidad en cada uno de los centros, se cumplen de acuerdo con los parámetros que las caracterizan. Para el transporte de los RFF de los centros de acopio interno a los externos, se utilizan los dos tipos de camiones con los que se cuentan, el camión de capacidad 10 ton., para enviar del centro interno 1 al externo 1 , del 3 al 2, del 3 al 3 y del 6 al 3 . El camión de 5 ton., se utiliza para cubrir las rutas de 2 a 1,4 y 1 y de 5 a 1 . Para cumplir con todos estos envíos, sólo se necesitó de un viaje entre cada origen y destino (tabla 6).

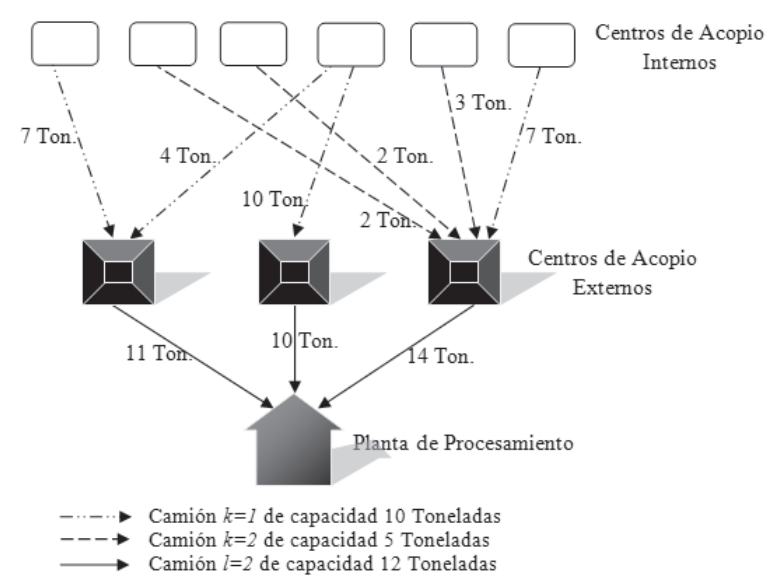

Figura 4. Resultados obtenidos al solucionar el modelo general. 
Tabla 6. Solución del modelo: Orígenes “i”, Destinos “j”, Cantidad enviada y Número de Viajes

\begin{tabular}{|c|c|c|c|c|c|c|}
\hline \multirow{2}{*}{$\begin{array}{l}\text { Acopios } \\
\text { internos i }\end{array}$} & \multicolumn{6}{|c|}{ Acopios externos j } \\
\hline & \multicolumn{2}{|c|}{1} & \multicolumn{2}{|c|}{2} & \multicolumn{2}{|l|}{3} \\
\hline \multirow{2}{*}{1} & $k=1$ & $k=2$ & $k=1$ & $k=2$ & $k=1$ & $k=2$ \\
\hline & 7 Ton1 Viaje & - & - & - & - & - \\
\hline \multirow{2}{*}{2} & $k=1$ & $k=2$ & $k=1$ & $k=2$ & $k=1$ & $k=2$ \\
\hline & - & 3 Ton 1 Viaje & - & - & - & - \\
\hline \multirow[b]{2}{*}{3} & $k=1$ & $k=2$ & $k=1$ & $k=2$ & $k=1$ & $k=2$ \\
\hline & - & - & $\begin{array}{c}10 \text { Ton } 1 \\
\text { Viaje }\end{array}$ & - & 4 Ton 1 Viaje & - \\
\hline \multirow{2}{*}{4} & $k=1$ & $k=2$ & $k=1$ & $k=2$ & $k=1$ & $k=2$ \\
\hline & - & 2 Ton 1 Viaje & - & - & - & - \\
\hline \multirow{2}{*}{5} & $k=1$ & $k=2$ & $k=1$ & $k=2$ & $k=1$ & $k=2$ \\
\hline & - & 2 Ton 1 Viaje & - & - & - & - \\
\hline \multirow{2}{*}{6} & $k=1$ & $k=2$ & $k=1$ & $k=2$ & $k=1$ & $k=2$ \\
\hline & - & - & - & - & 7 Ton 1 Viaje & - \\
\hline
\end{tabular}

El plan de envío obtenido muestra que la recolección del centro de acopio interno 3 se envía a dos distintos centros externos (2 y 3), lo cual hace que no se cumpla la condición single sourcing. Luego de recibir la carga de los puntos internos, los puntos externos proceden a enviar a la planta procesadora (tabla 7), de acuerdo con los resultados; para los envíos de los tres centros de acopio externo, sólo se utiliza el medio de transporte $I=2$ con capacidad de carga de 12 Ton.

Tabla 7. Solución del modelo: Orígenes “j”, Destinos “t”, Cantidad enviada y Número de Viajes

\begin{tabular}{|c|c|c|}
\hline Acopios Externos j & \multicolumn{2}{|c|}{ Planta Procesadora t } \\
\cline { 2 - 3 } & \multicolumn{2}{|c|}{$\mathbf{1}$} \\
\hline \multirow{2}{*}{1} & $I=1$ & $I=2$ \\
\cline { 2 - 3 } & - & 14 Ton.2 Viajes \\
\hline \multirow{2}{*}{2} & $I=1$ & $I=2$ \\
\cline { 2 - 3 } & - & 10 Ton. 1Viaje \\
\hline \multirow{2}{*}{3} & $I=1$ & $I=2$ \\
\cline { 2 - 3 } & - & 11 Ton. 1 Viaje \\
\hline
\end{tabular}

El modelo elaborado (tablas 6 y 7 ), se corrió en el software GAMS versión académica, a través de un computador portátil con procesador Intel Core 15. 
Análisis y Discusión de Resultados Modelo Single Sourcing. El modelo se elaboró en GAMS versión académica. Fue necesario recurrir al servicio del NEOS SERVER - SolverXpresMP, teniendo en cuenta que la versión académica no fue suficiente para realizar las corridas. El computador portátil utilizado tiene procesador Intel Core 15.

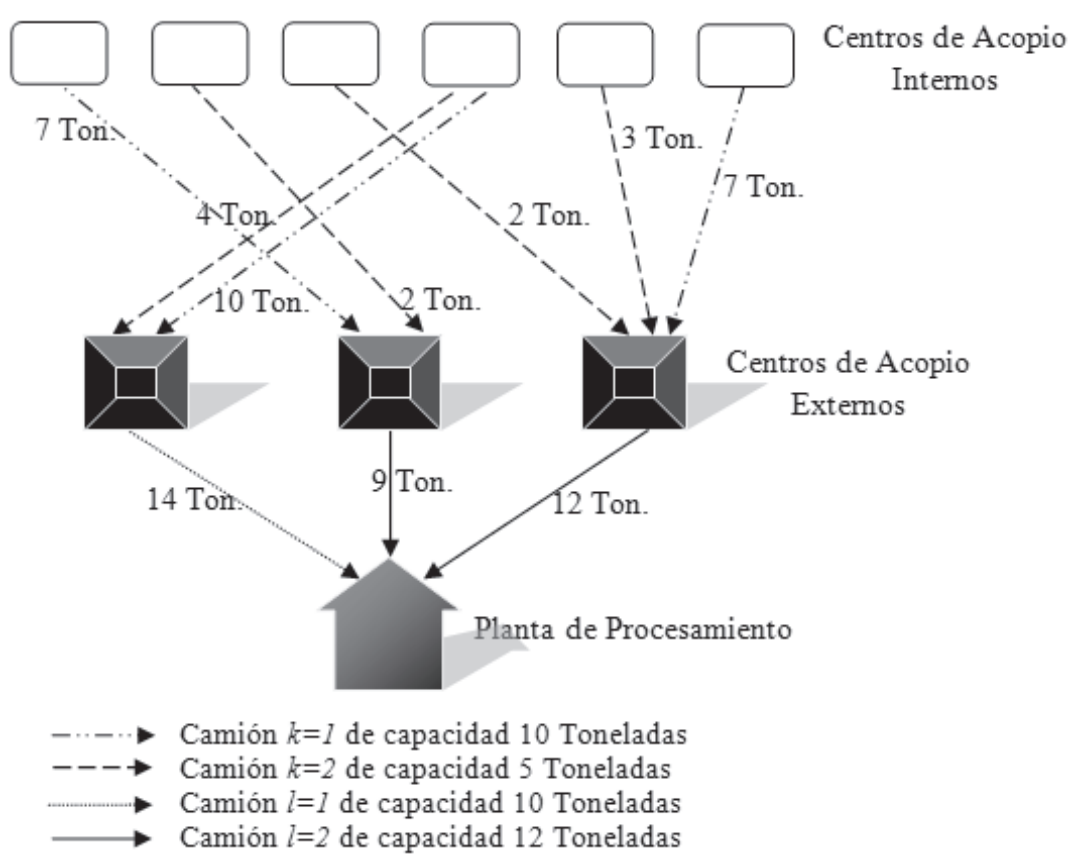

Figura 5. Resultados obtenidos al solucionar el modelo general con single sourcing

Para evaluar la formulación planteada, se utilizan los mismos parámetros establecidos en el modelo general. De una finca, sólo se puede enviar a un centro de acopio externo por razones técnicas, atendiendo que no se permite mezclas de los frutos, lo cual se puede dar sólo en el punto crossdock, después de un análisis fisicoquímico de control de calidad y seguimiento a proveedores y transportistas. La solución del modelo con la condición single sourcing, restringe la libertad de envíos entre un origen (centro de acopio interno), y múltiples destinos (centros de acopio externo). El modelo planteado mostró como resultado, un costo total por tonelada de $\$ 5.921,9$, manteniendo los mismos parámetros utilizados en el modelo general. A continuación, se presenta un gráfico que muestra un resumen de los resultados: La figura 5 muestra que cada uno de los centros de acopio interno envía únicamente a un centro de acopio externo. Los resultados de cantidad enviada, número de viajes y tipo de camión utilizado, se muestran en las tablas 8 y 9. 
Tabla 8. Resultados de la solución del modelo single sourcing: Orígenes “i”, Destinos “j”, Cantidad enviada y Número de Viajes

\begin{tabular}{|c|c|c|c|c|c|c|}
\hline \multirow{2}{*}{$\begin{array}{l}\text { Acopios } \\
\text { internos i }\end{array}$} & \multicolumn{6}{|c|}{ Acopios externos j } \\
\hline & \multicolumn{2}{|c|}{1} & \multicolumn{2}{|c|}{2} & \multicolumn{2}{|r|}{3} \\
\hline \multirow{2}{*}{1} & $k=1$ & $k=2$ & $k=1$ & $k=2$ & $k=1$ & $k=2$ \\
\hline & 7 Ton 1 Viaje & - & - & - & - & - \\
\hline \multirow{2}{*}{2} & $k=1$ & $k=2$ & $k=1$ & $k=2$ & $k=1$ & $k=2$ \\
\hline & - & 3 Ton 1 Viaje & - & - & - & - \\
\hline \multirow[b]{2}{*}{3} & $k=1$ & $k=2$ & $k=1$ & $k=2$ & $k=1$ & $k=2$ \\
\hline & - & - & & - & $\begin{array}{l}10 \text { Ton } \\
1 \text { Viaje }\end{array}$ & 4 Ton 1 Viaje \\
\hline \multirow{2}{*}{4} & $k=1$ & $k=2$ & $k=1$ & $k=2$ & $k=1$ & $k=2$ \\
\hline & - & 2 Ton 1 Viaje & - & - & - & - \\
\hline \multirow[b]{2}{*}{5} & $k=1$ & $k=2$ & $k=1$ & $k=2$ & $k=1$ & $k=2$ \\
\hline & - & - & - & $\begin{array}{l}2 \text { Ton } \\
1 \text { Viaje }\end{array}$ & - & - \\
\hline \multirow[b]{2}{*}{6} & $k=1$ & $k=2$ & $k=1$ & $k=2$ & $k=1$ & $k=2$ \\
\hline & - & - & $\begin{array}{c}7 \text { Ton } \\
1 \\
\text { Viaje }\end{array}$ & - & - & - \\
\hline
\end{tabular}

Tabla 9. Resultados de la solución del modelo single sourcing: Orígenes “j”, Destinos "t”, Cantidad enviada y Número de Viajes

\begin{tabular}{|c|c|c|}
\hline \multirow{2}{*}{$\begin{array}{c}\text { Acopios } \\
\text { Externos j }\end{array}$} & \multicolumn{2}{|c|}{ Planta Procesadora t } \\
\cline { 2 - 3 } \multirow{2}{*}{1} & $I=1$ & $I=2$ \\
\cline { 2 - 3 } & - & 12 Ton. 2 Viajes \\
\hline \multirow{2}{*}{2} & $I=1$ & $I=2$ \\
\cline { 2 - 3 } & - & 9 Ton. 1 Viaje \\
\hline \multirow{2}{*}{3} & $I=1$ & $I=2$ \\
\cline { 2 - 3 } & 14 Ton. 2 Viaje & - \\
\hline
\end{tabular}

\section{CONCLUSIONES}

El modelo matemático denominado de escenario general, se constituye en una herramienta que permite su uso en la gestión del transporte de RFF, articulando programación de cosecha en las fincas, programación de rutas (transportista de 
RFF), entre puntos internos y externos, transportes entre puntos externos y plantas, así como la programación de medios diferentes, incluido el uso de ventanas de tiempo.

La consideración de la aplicación de un escenario single sourcing entre centros de acopio internos y externos, como una medida de carácter administrativo para mejorar el funcionamiento, seguimiento y control del proceso en cuestión, trae consigo restricciones de carácter técnico que pueden impactar las condiciones de distribución del sistema en lo referente a cantidades de envío, número de viajes por desarrollar y medios por utilizar, así como también es posible que incremente el costo como un efecto de la reducción del espacio de soluciones factibles.

La importancia de plantear un modelo single sourcing para gestionar el transporte, genera versatilidad y distintas posibilidades para evaluar escenarios en donde se ven enfrentados a diario, los agentes que intervienen en este sector, buscando mecanismos de coordinación e integración, planteando actividades de control de calidad, combinaciones de carga, manejo de información, incorporación de nuevos medios de transporte, entre otros, todo lo cual implica una evaluación económica y técnica que establezca las incidencias de estas decisiones y que gracias al modelo propuesto, pueden ser evaluadas con diversos parámetros. Estos modelos surgen de la necesidad de los productores de contar con proveedores que puedan ser capaces de cumplir sus necesidades, entre ellas la de tener proveedores que puedan suministrar el servicio de transporte con un menor nivel de precios.

\section{REFERENCIAS BIBLIOGRÁFICAS}

[1] Consejo Nacional de Política Económica y Social (2007). Documento CONPES 3477. Estrategia para el Desarrollo Competitivo del Sector Palmero Colombiano. Bogotá: Departamento Nacional de Planeación.

[2] Cuao L., Angélica. (2009). Estudio de las condiciones operativas en el proceso finca-extractora de la palma africana en el departamento de Magdalena. Santa Marta: Universidad Sergio Arboleda, Escuela de Negocios.

[3] FEDEPALMA 2008. Anuario estadístico, Bogotá. FEDEPALMA.

[4] Duarte y Guterman. (2009). Informe de actualización de costos de producción del aceite de palma. Bogotá, FEDEPALMA.

[5] Corley, R H V. y Law, I H. (2001). Ripening, harvesting and oil extraction. En: The planter, vol 77. pp. 507-524. 
[6] Jerez, J. y Amézquita, M. (2004). Transporte de fruta en cajas en UNIPALMA S.A. En: Revista Palmas. Vol. 25, No. Especial 2, pp. 472-175.

[7] Fontanilla, C.; Pachón, S.; Castiblanco, J.; Mosquera, M. y Sanchez, A. (2010). Referenciación competitiva a los sistemas de evacuación y alce de fruto. En: Area 51 Publicidad y Comunicación, p. 48.

[8] Fontanilla, C. y Castiblanco, J. (2009). Cable vía en la cosecha de la palma de aceite. En: Revista Palmas, vol 30, No 4, pp. 53-64.

[9] Ademosun, O. C. (1982). Location-allocation models for oil palm production in Nigeria based on the feasible set approach. En: International Journal of production Research. Vol. 20, No. 2, pp.211-226.

[10] Mosquera M., Valenzuela J., (2006). Estudio de logística para el proceso de transporte fruto de aceite en Colombia. En: Revista Palmas. Vol. 27, No. 4, pp. 55-64.

[11] García R., Martínez, M. y Palacios F., (2007). Tactical and operative optimization of the supply chain in the oil palm industry. Tesis Doctoral. Bogotá. Universidad de los Andes.

[12] Tinsay, E. (2007). Introducción a la red logística en el aceite de palma. En: Revista Palmas. Vol. 28, No. Especial, pp. 321-327.

[13] Yandra; Marimin; Jamaran, I; Eriyatno. y Tamura, H. (2007). An integration of multi-objective genetic algorithm and fuzzy logic for optimization of agroindustrial supply chain design. En: Proceedings of the $51^{\text {st }}$ Annual meeting of the ISSS. Tokyo.

[14] Ibrahim S., (2008). Transportation optimization model of palm oil products for northern peninsular Malaysia. Doctoral dissertation. Universiti Sains Malaysia.

[15] Gutierrez (2008). Planificación estratégica del biodiesel en Colombia. En: Book of Extended Abstracts. XIV Latin Ibero-American Congress on Operation Research (CLAIO).

[16] Sauian (2008). OR in Agriculture: The Use of Assignment Model. Proceedings of the Third Meeting of the EURO Working Group on Operational Research $(\mathrm{OR})$ in Agriculture and Forest Management (EWG-ORAFM).

[17] Fontanilla C., Adarme W., and Arango M., (2010). Programming tools, an alternative to optimize the logistics at the transportation process of the oil palm 
fresh fruit bunches (FFB). En: Operations and Technology Management, vol 13: Innovative methods in Logistics, Emerging trends, concepts and technologies. Hamburg, Alemania. 
